\title{
Evaluating Potentially Modifiable Risk Factors for Prevalent and Incident Nocturia in Older Adults
}

\author{
Theodore M. Johnson II, MD, MPH, ${ }^{* \dagger}$ Richard W. Sattin, MD,, $\mathcal{S}$ Patricia Parmelee, PhD, ${ }^{* \neq}$ Nancy $H$. \\ Fultz, PhD," and Joseph G. Ouslander, MD* ${ }^{* \neq}$
}

OBJECTIVES: To examine associations between nocturia and potentially modifiable risk factors in older adults.

DESIGN: Secondary analysis of cross-sectional and longitudinal data.

SETTING: Respondents were selected using populationbased sampling, drawing from a single Michigan county in 1983. They were followed through 1990.

PARTICIPANTS: Community-living adults aged 60 and older.

MEASUREMENTS: Episodes of nocturia, development of nocturia at 2 years after baseline survey, age, sex, hypertension, diabetes mellitus, drinking fluids before bedtime, amount of fluid intake before bedtime, diuretic use, and 24-hour coffee intake. All measures were self-reported.

RESULTS: Bivariate cross-sectional analysis revealed significant associations with two or more episodes of nocturia for hypertension (odds ratio $(\mathrm{OR})=1.7,95 \%$ confidence interval $(\mathrm{CI})=1.37-2.1)$, diabetes mellitus $(\mathrm{OR}=1.51$, $95 \% \mathrm{CI}=1.1-2.0)$, diuretic use $(\mathrm{OR}=1.7,95 \% \mathrm{CI}=1.3-$ $2.1)$, age $(\mathrm{OR}=1.05$ per additional year over $60,95 \%$ 1.03-1.06), and number of cups of coffee (OR $=0.93$ for each cup of coffee, $95 \% \mathrm{CI}=0.89-0.97)$. In multivariate analysis, hypertension $(\mathrm{OR}=1.52,95 \% \mathrm{CI}=1.2-1.9)$, diuretic use $(\mathrm{OR}=1.3,95 \% 95 \% \mathrm{CI}=1.0-1.7)$, and age $(\mathrm{OR}=1.04$ per additional year over $60,95 \% 1.03-1.06)$

From the *Birmingham/Atlanta Veterans Affairs Geriatric Research, Education, and Clinical Center, Atlanta, Georgia; 'Emory Center for Health in Aging, Atlanta, Georgia; ${ }^{\ddagger}$ Division of Geriatric Medicine and Gerontology, and Wesley Woods Center of Emory University, Emory

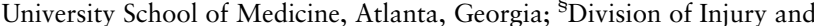
Disability Outcomes and Programs, National Center for Injury Prevention and Control, Centers for Disease Control and Prevention, Atlanta, Georgia; and "Institute for Research on Women and Gender, University of Michigan, Ann Arbor, Michigan.

Drs. Johnson and Parmelee received support from the Emory Center for Health in Aging. Dr. Fultz received support from the Building Interdisciplinary Research Careers in Women's Health Program of the National Institutes of Health (K12 HD01438). This paper was presented in poster form at the 2004 Annual Congress of the International Continence Society \& International Uro-Gynecologic Association, Paris, France.

Address correspondence to Theodore M. Johnson II, Birmingham/Atlanta GRECC, 508/11B, Atlanta VAMC, 1670 Clairmont Road, Decatur, GA 30033. E-mail: Ted.Johnson@med.va.gov

DOI: $10.1111 / \mathrm{j} .1532-5415.2005 .53321 . x$ were independently associated with two or more nocturia episodes per night. No baseline factors predicted future development of nocturia (save for age, in one model).

CONCLUSION: Hypertension, older age, and diuretic use were independently associated with two or more episodes of nocturia in cross-sectional analysis. No baseline factor was related to the development of nocturia over a 2-year interval in this sample. Nighttime fluid intake and coffee intake, practices providers commonly target in patients with nocturia, were not associated with nocturia in this population-based sample of community-living older adults. J Am Geriatr Soc 53:1011-1016, 2005.

Key words: nocturia; aging; fluid intake; hypertension; epidemiology

$\mathbf{N}$ octuria, defined as waking at night to void, ${ }^{1}$ is common $^{2}$ and occurs with increasing frequency with advancing age. For older adults, nocturia is a significant cause of sleep disruption ${ }^{3}$ and is associated with double the risk of falling. ${ }^{4}$ Nocturia has been rated as one of the most bothersome lower urinary tract symptoms. ${ }^{5,6}$

Often, practitioners recommend to their patients behavioral modifications (drink less water at night, reduce coffee) or focus on managing certain conditions (improving diabetes mellitus control) to treat nocturia, but whether such potentially modifiable factors are associated with nocturia is uncertain. Population-based studies on nocturia ${ }^{7-14}$ have largely been inventories of urological symptoms. Although these studies provide prevalence estimates, they provide limited insight into the epidemiology of nocturia. Clinic-based populations have many clinical details on the participants, 2,15,16 yet these referral-based samples are not representative of the general population, limiting generalizability. There are few longitudinal studies of voiding symptoms. ${ }^{17}$ No study has examined, in older adults, whether potentially modifiable risk factors for nocturia predict future development of nocturia.

The purpose of these analyses was to examine crosssectional and longitudinal data to determine whether several potentially modifiable risk factors were associated with 
nocturia. The a priori hypothesis was that specific risk factors presumed to be related to nocturia, such as nighttime fluid intake and hypertension, would be significantly associated with the presence and development of nocturia.

\section{METHODS}

Data from the Medical, Epidemiologic, and Social aspects of Aging (MESA) Study, a population-based, community sample of noninstitutional adults aged 60 and older, initially collected in 1983, were used in these analyses. The institutional review board of the University of Michigan approved the MESA study. Trained interviewers conducted in-person interviews with 1,956 participants in 1983/84 (baseline). Additional data were collected in 1984/85 $(\mathrm{n}=1,334$, Year 1 follow-up), 1985/86 $(\mathrm{n}=1,289$, Year 2 follow-up), and 1989/90 ( $\mathrm{n}=1,199$, Year 6 follow-up). Although the baseline and Year 2 follow-up data were the source of the longitudinal results reported in this paper, the Year 1 and Year 6 follow-up data were also examined. These data have been particularly valuable because of the detailed questioning used to describe the epidemiology of voiding problems in general and urinary incontinence in particular. ${ }^{18-24}$ For this secondary analysis, participants' anonymity was guaranteed through the use of an investigator agreement, and all direct identifiers were removed from the database.

For the nocturia variable, the question, "Generally, how many times do you usually urinate after you have gone to sleep at night?" was used. The wording of this question is fairly consistent with the International Continence Society definition of nocturia published in $2002,{ }^{1}$ except that an interviewer instruction not read aloud told the interviewer to include nighttime urinary incontinence if mentioned. Because of a slight change in the wording of the nocturia question (from "How many times do you usually urinate after you have gone to sleep?" in baseline and Years 1 and 2 follow-up surveys to "usually urinate after you have fallen asleep at night"), Year 6 follow-up data were not used for any longitudinal analyses.

Nocturia was dichotomized into two or more (nocturia) and less than two (no nocturia); an alternative cutpoint at three or more and less than three was also examined. Where nocturia was managed as an ordinal variable in additional analyses, persons reporting five or more episodes were collapsed into a category "five or more episodes."

To be categorized as having incident nocturia, a participant must have reported zero or one episode of nocturia per night at baseline and reported two or more episodes of nocturia at the 2-year follow-up. Alternative coding strategies for incident nocturia were also explored: (1) those with zero or one episode of nocturia at baseline would be considered incident if they had an increase of two or more additional episodes; and (2) those with zero episodes of nocturia at baseline would be considered incident with any future episode of nocturia at follow-up.

Variables suggested in the literature as contributing to or causing nocturia were included in the analyses; each additional year of age over 60, taking fluid intake at night (percentage of an 8-ounce serving), and number of cups of coffee in a 24-hour period (all continuous variables); being told by a doctor that you have hypertension, being told by a doctor that you have diabetes mellitus, being male, drinking fluids at night, and taking a diuretic (all dichotomous variables). Coffee drinking was recorded as total number of cups per day but not as caffeinated or decaffeinated.

Following the a priori analyses just described, correlational analyses were performed of nocturia reports at each time point (baseline, Year 1 follow-up, and Year 2 followup). Additional analyses of the correlation of nocturia reports over time were performed, restricting the sample to (1) those with higher levels of education and only those with zero episodes or three or more episodes of nocturia. An additional cross-sectional analysis of Year 6 follow-up data was used to attempt to reproduce cross-sectional multivariate analysis performed on baseline data.

SPSS 11.0 was used for all analyses (SPSS, Inc., Chicago, IL). Variables were evaluated for coding errors by inspecting range, mean, and median values. To examine for collinearity between the variables, a correlational analysis was used. Effect modification by age or sex of each variable with respect to nocturia was examined. Variables related in bivariate analysis to nocturia with $P<.10$ were further evaluated in backwards stepwise multivariable logistic regression. The criterion for inclusion in the model was $P<.05$, with a variable withdrawn if $P>.10$. If no significant associations were found in bivariate analysis, no multivariate analyses were performed. Models were repeated with the previously described alternative coding strategies for nocturia and incident nocturia.

\section{RESULTS}

Figure 1 shows the progression of study participants from baseline through Year 2 follow-up and their responses to nocturia questions. Of the original 1,956 MESA participants at the baseline survey, 1,632 (83.4\%) answered the question on nocturia (Table 1). Of these 1,632 participants, $31 \%, 37 \%, 20 \%, 8 \%, 3 \%$, and $1 \%$ reported zero, one, two, three, four, and five or more episodes of nocturia, respectively. As for the collapse of the highest categories, only $1 \%$ of those answering the nocturia question had six or more episodes of nocturia. For respondents by age and sex at baseline, in men aged 60 to 69,70 to 79 , and 80 and older, $25.6 \%, 37.7 \%$, and $57.1 \%$ reported two or more episodes of nocturia, respectively. In women aged 60 to 69 , 70 to 79 , and 80 and older, $26.9 \%, 35.5 \%$, and $42.3 \%$ had two or more episodes of nocturia, respectively.

By Year 2 follow-up, there were 1,289 respondents (66\% of the original sample). At Year 2 follow-up, 99.2\% $(\mathrm{n}=1,279)$ of the sample answered the nocturia question. Of these 1,279 respondents, $28 \%, 39 \%, 19 \%, 10 \%, 3 \%$, and $2 \%$ reported zero, one, two, three, four, and five or more episodes of nocturia, respectively. Of the 748 Year 2 follow-up respondents who reported zero or one nocturia episodes at baseline, $259(34.6 \%)$ reported two or more episodes of nocturia (incident nocturia). Of those who reported two or more episodes of nocturia at baseline and answered the nocturia question at Year 2 follow-up $(n=357), 237(66.4 \%)$ reported zero or one episode of nocturia (remitting nocturia). Because there was no effect on the results of placing the cutpoint at three, only the results of the cutpoint at two have been reported. 


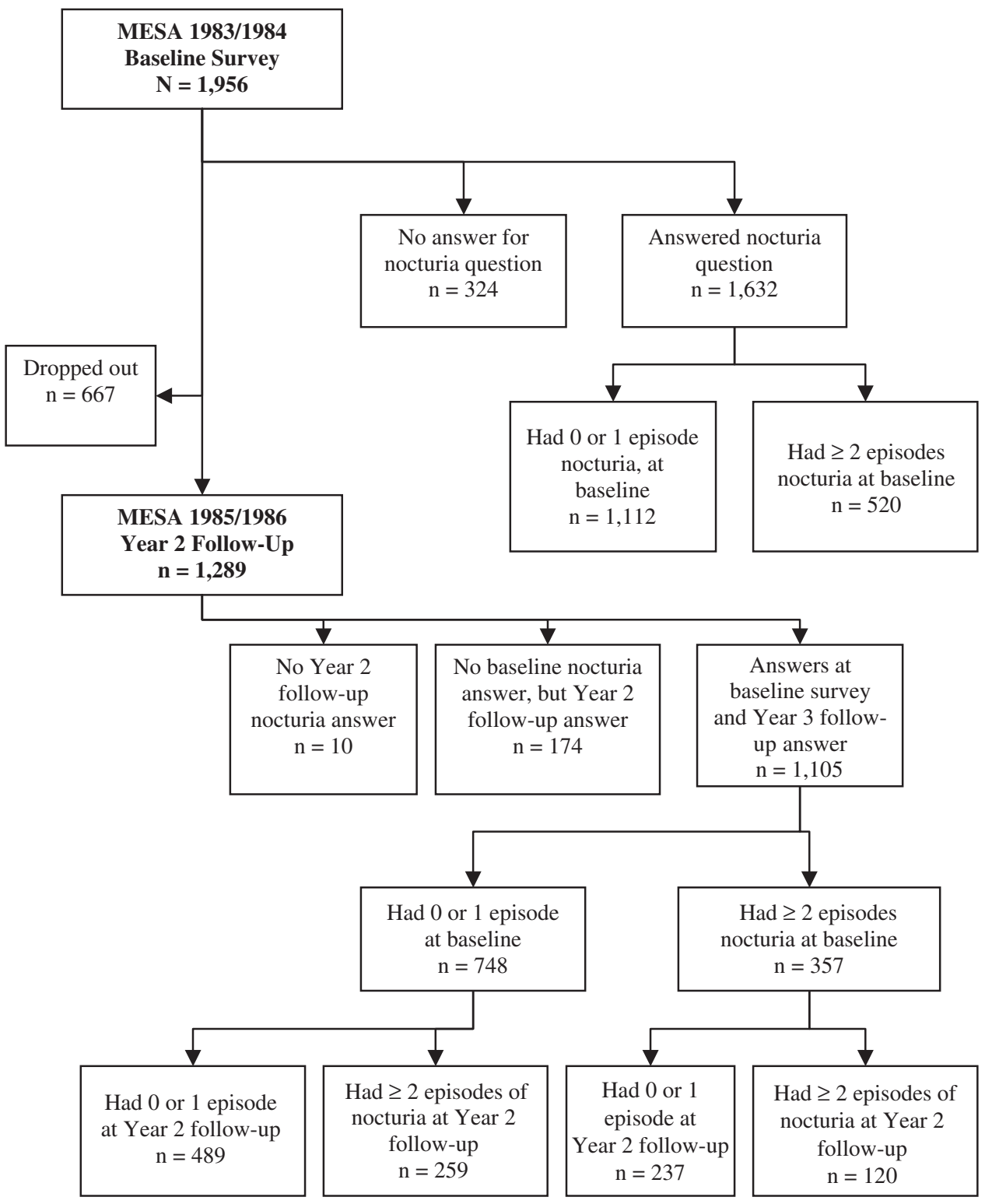

Figure 1. Flow chart of participants in the Medical, Epidemiologic, and Social aspects of Aging (MESA) Study. Chart shows how participants in baseline and follow-up survey were tracked through waves. Ratio for incident nocturia calculated by taking $\mathrm{N}$ of "Having two or more episodes of nocturia at Year 2 follow-up" $(\mathrm{n}=259)$ and dividing by "Had 0 or 1 nocturia episode at baseline" $(\mathrm{n}=748)$.

The initial 1,956 members of the sample did not differ significantly from subjects at Year 1 follow-up or Year 2 follow-up with respect to age at baseline or sex (analysis not shown). There was a difference in mean age, but not sex, between nonresponders and responders. No important collinearity between the variables was detected, and no effect modification by age or sex was found for any variable relevant to nocturia.

Table 2 illustrates the results from bivariate and multivariate analyses of risk factors for nocturia. For the multivariate analyses, only variables retained in the final model have odds ratios reported.

No baseline variables examined (male sex, hypertension, diabetes mellitus, bedtime fluids/amount of bedtime fluids, diuretic use, age, coffee intake) were associated with incident nocturia at 2 years. All point estimates for these bivariate odds ratios were between 0.88 and 1.02 . Alternative codings of nocturia $(\geq 3,<3)$ and a different length of follow-up ( 1 year) were also employed, with little difference in the results except when incident nocturia was coded as going from zero episodes of nocturia to one or more episodes. Using that categorization, age was statistically associated with incident nocturia at 1 year (odds ratio $(\mathrm{OR})=1.03,95 \%$ confidence interval $(\mathrm{CI})=1.00-1.07)$ but not 2 years $(\mathrm{OR}=0.98,95 \% \mathrm{CI}=0.95-1.01)$. Because no coding yielded significant associations in bivariate analyses (other than age in one model), no multivariate analyses for incident nocturia were performed.

There were several additional analyses performed post hoc, as described in the Methods section; these results are 
Table 1. Baseline Characteristics of the Study Population

\begin{tabular}{|c|c|c|c|c|}
\hline \multirow[b]{2}{*}{ Characteristic } & \multicolumn{2}{|c|}{ Episodes of Nocturia } & \multirow{2}{*}{$\begin{array}{l}\text { Respondents to } \\
\text { Nocturia Question } \\
(n=1,632)\end{array}$} & \multirow{2}{*}{$\begin{array}{l}\text { Nonrespondents to } \\
\text { Nocturia Question } \\
\quad(n=324)\end{array}$} \\
\hline & $\begin{array}{c}0 \text { or } 1 \\
(n=1,112)\end{array}$ & $\begin{array}{c}\geq 2 \\
(n=520)\end{array}$ & & \\
\hline & 68.9 & $71.5^{\dagger}$ & 69.7 & $71.5^{\ddagger}$ \\
\hline & 40.6 & 41.0 & 40.7 & 42.6 \\
\hline at bedtime, ounces & 10.0 & 10.7 & 10.2 & - \\
\hline er day & 2.8 & $2.4^{*}$ & 2.7 & - \\
\hline & 28.2 & $39.7^{\dagger}$ & 31.9 & - \\
\hline t bedtime, \% & 77.0 & 76.6 & 76.9 & - \\
\hline at you have diabetes mellitus, \% & 12.4 & $17.6^{*}$ & 14.2 & - \\
\hline at you have hypertension, \% & 44.0 & $57.1^{\dagger}$ & 48.2 & - \\
\hline
\end{tabular}

$P<{ }^{*} .05,{ }^{\dagger} .001$ for difference between those with nocturia and those without nocturia.

${ }^{\ddagger} P<.001$ for difference between respondents to nocturia and nonrespondents to nocturia question.

$-=$ percentages suppressed because $\mathrm{n} \leq 10$ for these categories.

enumerated here. Nocturia reports at different waves were negligibly correlated. Pearson correlations were 0.02 (baseline and Year 1 follow-up), -0.02 (baseline and Year 2 follow-up), and 0.08 (Year 1 follow-up and Year 2 followup); only Year 1 and Year 2 follow-up reports were significantly correlated ( $\mathrm{n}=1,265, P=.05)$. Restricting the sample to those with a high school education or greater made little substantive difference in the correlations. The Pearson correlations were -0.04 (baseline and Year 1 follow-up), -0.02 (baseline and Year 2 follow-up), and 0.17 (Year 1 follow-up and Year 2 follow-up - a statistically significant correlation $P<.05$ ).

These correlations were repeated, restricting the sample to respondents who had zero or three or more episodes of nocturia. At baseline and Year 1 and Year 2 followup, there were 498, 352, and 401 participants who had zero episodes and 199, 195, and 158 who had three or more episodes of nocturia, respectively. Between Year 1 and Year 2 follow-up, those who reported three or more episodes of nocturia were significantly more likely $\left(\chi^{2} P=.048\right)$ to report three or more episodes of nocturia at Year 2 follow-up. All other comparisons between waves were nonsignificant.

Repeating the baseline multivariable analysis using Year 6 follow-up data yielded slightly different results. With baseline data, being told that you have hypertension, reporting diuretic use, and age were independently significant in multivariate analyses. Using Year 6 follow-up data and beginning with the same set of variables (age, hypertension, diabetes mellitus, coffee drinking, and reporting diuretic use), a slightly different model resulted. For Year 6 followup data, age $(\mathrm{OR}=1.04,95 \% \mathrm{CI}=1.02,1.06)$, diuretic use $(\mathrm{OR}=1.58,95 \% \mathrm{CI}=1.21,2.07)$, and diabetes mellitus $(\mathrm{OR}=1.52,95 \% \mathrm{CI}=1.06,2.17)$ were related to reporting two or more episodes of nocturia.

\section{DISCUSSION}

In this population-based sample of community-living adults aged 60 and older, hypertension, advanced age, and diuretic use were independently associated with having two or more episodes of nocturia in cross-sectional analysis. Year 6

Table 2. Factors Associated in Cross-Sectional Bivariate and Multivariate Analyses with Having Two or More Episodes of Nocturia

Bivariate Analysis* Multivariate Analysis ${ }^{\dagger}$

Factor Odds Ratio (95\% Confidence Interval)

Dichotomous variables

Being male

Being told by a doctor that you have hypertension

Being told by a doctor that you have diabetes

Having fluids at bedtime

Using a diuretic

Odds Ratio (95\% Confidence Interval)

Continuous variables

Age, in years

Amount of fluids at bedtime (\% of an 8-ounce cup) Number of cups of coffee in a 24-hour period

$1.02(0.82-1.26)$
$1.70(1.38-2.10)$
$1.51(1.13-2.01)$
$0.97(0.76-1.25)$
$1.67(1.34-2.09)$
$1.05(1.03-1.06)$
$1.05(0.96-1.15)$
$0.93(0.89-0.97)$

$1.52(1.19-1.94)$

$1.31(1.01-1.69)$

$1.04(1.03-1.06)$

\footnotetext{
* Maximum $\mathrm{n}=1,632$ for each bivariate analysis based upon missing values in nocturia response.

${ }^{\dagger} \mathrm{n}=1,563$ for this multivariate analysis.
} 
follow-up data (but not baseline) showed an independent association between diabetes mellitus and nocturia. Total 24-hour coffee drinking was associated with reporting less nocturia in bivariate analysis. Despite the fact that practitioners often encourage decreased nighttime fluid drinking, no measure of nighttime fluid intake in this sample was associated with nocturia. In longitudinal analyses, no baseline factor was related to the development of nocturia over a 2-year interval. An epidemiological framework was used for these analyses, based on the reasoning that, if a potentially modifiable risk factor were shown to be associated with nocturia or incident nocturia, it would be a reasonable factor on which to intervene. Alternatively, if no such association were found, it might not be a reasonable target.

These results are congruent with other studies. Hypertension has previously been shown to be associated with nocturnal polyuria and nocturia. ${ }^{25,26}$ Hypertension might be related to nocturia by its effect on cardiovascular physiology (edema, congestive heart failure with atrial stretch and release of atrial natriuretic peptide) or renal physiology (renal effects on glomerular filtration and tubular transport). Hypertension may cause resetting of the pressurenatriuresis relation in the kidney. ${ }^{26}$ Diabetes mellitus affects renal physiology. In the short term, poorly controlled diabetes mellitus causes glucosuria and polyuria. (Although this would cause polyuria in the day and night and not specifically nocturnal polyuria.)

Although not previously described, an association between diuretic use and nocturia is biologically plausible. Diuretic use may be related to nocturia because of its relationship with hypertension, congestive heart failure, or peripheral edema. Alternatively, participants may have used diuretics in the afternoon as a treatment for nocturia. ${ }^{27} \mathrm{Had}$ diuretic use predicted the future development of nocturia, the temporal relationship between diuretic use and nocturia would be more certain.

These results did not support some commonly held beliefs. More than $65 \%$ of all participants (with and without nocturia) reported bedtime fluid intake. Neither bedtime fluid intake (as a yes or no variable) nor amount of fluid drunk before bedtime was associated with nocturia. This finding is consistent with accumulated anecdotal patient reports. For most patients, fluid restriction frequently does not work, except in cases in which patients clearly related that they intentionally drank a large quantity of fluid late in the evening before retiring (personal communication, Jeffrey Weiss, July 14, 2004). When those patients ceased drinking after supper, they tended to improve, if only a little. Thus, reducing evening or bedtime fluid intake may best be regarded as targeted advice for certain patients as opposed to general advice for all. No studies could be identified linking nighttime fluid discontinuation to nocturia reduction, although this recommendation appears in guidelines. ${ }^{1}$ Most patients with nocturia have reasoned that it is a good idea to stop drinking before bedtime; so further instructions along these lines are frequently unhelpful. Because many patients may have fluid intake barely sufficient to meet their physiologic needs - as well as related problems such as constipation - advice to restrict fluids has potential to cause more harm than benefit for some patients. Data on 24-hour fluid intake showed no association between 24-hour intake and nocturia. With regard to coffee use, coffee drinking over a 24-hour period reduced odds of nocturia in bivariate analysis. These analyses would not support restricting overall coffee usage to reduce nocturia. Morning coffee use could act as a daytime diuretic and therefore reduce nighttime voids.

Longitudinal analyses in this sample failed to demonstrate any baseline risk factors that predicted future development of nocturia. Further investigation into this puzzling result showed that reporting nocturia was not stable over time in this study. Between baseline and Year 2 follow-up, more than $30 \%$ of the sample who initially reporting having fewer than two episodes of nocturia 2 years later reported having two or more episodes. In addition, $60 \%$ of those with two or more episodes at baseline reported fewer than two episodes by Year 2 follow-up.

These findings called into question the stability of the answer to the nocturia question and how stable nocturia is as a symptom. There are four lines of evidence suggesting that the nocturia question used in this survey produced accurate answers. First, the question used here has reasonable face validity and matches well with the International Continence Society definition. ${ }^{1}$ Second, age- and sex-specific prevalence data on nocturia derived here match prevalence estimates from a recently published study derived from a population-based sample of 92,491 respondents. ${ }^{28}$ Third, baseline cross-sectional analyses give similar results to the Year 6 follow-up cross-sectional analyses. Fourth, when considering only those with nocturia reports at the extremes (those with no nocturia and those with $\geq 3$ episodes), there was greater stability of these data.

Although many individuals gave markedly different answers to the nocturia question over time, the mean response of the group changed little. Considering baseline and Year 2 follow-up samples to be two sets of cross-sectional data, for example, the data appear much more stable; the prevalence of nocturia of two or more episodes for those aged 60 and older (baseline) is $32.3 \%$, and the prevalence for those aged 62 and older (Year 2 follow-up) is 34.3\%. Considered this way, the 2-year incidence appears to be $2 \%$.

There are additional limitations to these analyses. The lack of specificity of certain questions limits interpretation. Participants answered questions about coffee intake but did not specify whether the coffee was decaffeinated or whether they drank it in the morning or afternoon. Additionally, other sources of caffeine in the diet or in over-the-counter medications could not be assessed. Participants spoke about fluids before bedtime but gave no information about the length of the interval between intake and bedtime. As for diuretic use, these data are not specific as to the medical indication for diuretic use. Use may have been for hypertension management, edema states, or congestive heart failure; for any individual, the indication for a diuretic may have been different in 1984 as opposed to 2005. As for the diagnoses of hypertension and diabetes mellitus, there were likely some misclassifications. Participants may have believed they had hypertension or diabetes mellitus but did not, or may have had it but were not yet aware. Several aspects of hypertension and diabetes mellitus management have changed significantly over the past 20 years. The diagnostic criteria now used for diabetes mellitus or hypertension result in more individuals being diagnosed with these conditions today than in the 1980 s. $^{29,30}$ For diabetes 
mellitus and hypertension, new pharmaceuticals have been developed over the past 20 years (e.g., for hypertension, calcium-channel blockers and angiotensin-converting enzyme inhibitors; for diabetes mellitus, biguanides and glitazones). The effect of these diagnostic and therapeutic changes in relation to findings presented here is unclear.

Despite these limitations, these findings add to the literature on nocturia. These data are consistent with previous reports demonstrating an association between hypertension and nocturia. For the clinician, the lack of association between nocturia and nighttime fluid intake or total coffee intake is noteworthy. This suggests that providers who routinely advocate fluid management interventions for nocturia should reassess whether this strategy benefits their patients.

\section{ACKNOWLEDGMENTS}

The authors thank Nancy Bond for her assistance in the preparation of the manuscript. Drs. Don Bliwise, Nancy Kutner, Michael Kutner, Yohannes Endeshaw, and Kathy Parker provided valuable feedback during early planning meetings. Dr. Michael Kutner provided critique of statistical analyses used. The late Dr. Regula Herzog gave freely of her time, offering advice and encouragement; she is missed.

\section{REFERENCES}

1. van Kerrebroeck P, Abrams P, Chaikin D et al. The standardisation of terminology in nocturia: Report from the Standardisation Sub-committee of the International Continence Society. Neurourol Urodyn 2002;21:179-183.

2. Homma Y, Imajo C, Takahashi S et al. Urinary symptoms and urodynamics in a normal elderly population. Scand J Urol Nephrol Suppl 1994;157:27-30.

3. Middelkoop HA, Smilde-van den Doel DA, Neven AK et al. Subjective sleep characteristics of 1,485 males and females aged 50-93: Effects of sex and age, and factors related to self-evaluated quality of sleep. J Gerontol A Biol Sci Med Sci 1996;51A:M108-M115.

4. Stewart RB, Moore MT, May FE et al. Nocturia: A risk factor for falls in the elderly. J Am Geriatr Soc 1992;40:1217-1220.

5. DuBeau CE, Yalla SV, Resnick NM. Implications of the most bothersome prostatism symptom for clinical care and outcomes research. J Am Geriatr Soc 1995;43:985-992.

6. Naughton MJ, Wyman JF. Quality of life in geriatric patients with lower urinary tract dysfunction. Am J Med Sci 1997;314:219-227.

7. Blanker MH, Bohnen AM, Groeneveld FP et al. Normal voiding patterns and determinants of increased diurnal and nocturnal voiding frequency in elderly men. J Urol 2000;164:1201-1205.

8. Malmsten UG, Milsom I, Molander U et al. Urinary incontinence and lower urinary tract symptoms: An epidemiological study of men aged 45 to 99 years. J Urol 1997;158:1733-1737.
9. Roberts RO, Jacobsen SJ, Jacobson DJ et al. Natural history of prostatism: High American Urological Association Symptom scores among communitydwelling men and women with urinary incontinence. Urology 1998;51: 213-219.

10. Sagnier PP, MacFarlane G, Richard F et al. Results of an epidemiological survey using a modified American Urological Association symptom index for benign prostatic hyperplasia in France. J Urol 1994;151:1266-1270.

11. Asplund R, Aberg H. Health of the elderly with regard to sleep and nocturnal micturition. Scand J Prim Health Care 1992;10:98-104.

12. Pinnock C, Marshall VR. Troublesome lower urinary tract symptoms in the community: A prevalence study. Med J Aust 1997;167:72-75.

13. Schatzl G, Temml C, Schmidbauer J et al. Cross-sectional study of nocturia in both sexes: Analysis of a voluntary health screening project. Urology 2000; 56:71-75.

14. Schmidbauer J, Temml C, Schatzl G et al. Risk factors for urinary incontinence in both sexes. Analysis of a health screening project. Eur Urol 2001;39: $565-570$

15. Kaplan SA, Bowers DL, Te AE et al. Differential diagnosis of prostatism: A 12year retrospective analysis of symptoms, urodynamics and satisfaction with therapy. J Urol 1996;155:1305-1308.

16. Teh GC, Sahabudin RM, Lim TC et al. Prevalence of symptomatic BPE among Malaysian men aged 50 and above attending screening during prostate health awareness campaign. Med J Malaysia 2001;56:186-195.

17. Lee AJ, Russell EB, Garraway WM et al. Three-year follow-up of a community-based cohort of men with untreated benign prostatic hyperplasia. Eur Urol 1996;30:11-17.

18. Diokno AC, Brock BM, Herzog AR et al. Medical correlates of urinary incontinence in the elderly. Urology 1990;36:129-138.

19. Diokno AC, Brown MB, Herzog AR. Relationship between use of diuretics and continence status in the elderly. Urology 1991;38:39-42.

20. Diokno AC, Brown MB, Goldstein NG et al. Urinary flow rates and voiding pressures in elderly men living in a community. J Urol 1994;151:1550-1553.

21. Fultz NH, Herzog AR. Epidemiology of urinary symptoms in the geriatric population. Urol Clin North Am 1996;23:1-10.

22. Herzog AR, Fultz NH. Prevalence and incidence of urinary incontinence in community-dwelling populations. J Am Geriatr Soc 1990;38:273-281.

23. Herzog AR, Diokno AC, Brown MB et al. Two-year incidence, remission, and change patterns of urinary incontinence in noninstitutionalized older adults. J Gerontol 1990;45:M67-M74.

24. Herzog AR, Diokno AC, Brown MB et al. Urinary incontinence as a risk factor for mortality. J Am Geriatr Soc 1994;42:264-268.

25. Goldman N, Lin IF, Weinstein M et al. Evaluating the quality of self-reports of hypertension and diabetes. J Clin Epidemiol 2003;56:148-154.

26. McKeigue PM, Reynard JM. Relation of nocturnal polyuria of the elderly to essential hypertension. Lancet 2000;355:486-488.

27. Reynard JM, Cannon A, Yang Q et al. A novel therapy for nocturnal polyuria: A double-blind randomized trial of frusemide against placebo. $\mathrm{Br} \mathrm{J}$ Urol 1998;81:215-218.

28. McGrother CW, Donaldson MM, Shaw C et al. Storage symptoms of the bladder: Prevalence, incidence and need for services in the UK. BJU Int 2004; 93:763-769.

29. American Diabetes A. Screening for type 2 diabetes. Diabetes Care 2003; 26(Suppl. 1):S21-S24.

30. Chobanian AV, Bakris GL, Black HR et al. Seventh report of the Joint National Committee on Prevention, Detection, Evaluation, and Treatment of High Blood Pressure. Hypertension 2003;42:1206-1252. 